\title{
Invasive mucinous adenocarcinoma of the lung
}

\author{
Lu Xü ${ }^{1 \#}$, Chenghui $\mathrm{Li}^{2,3 \#}$, Hongyang $\mathrm{Lu}^{3,4}$ \\ ${ }^{1}$ Department of Medical Oncology, The First People's Hospital Yongkang, Yongkang 321300, China; ${ }^{2}$ The Second Clinical Medical College, \\ Zhejiang Chinese Medical University, Hangzhou 310053, China; ${ }^{3}$ Department of Thoracic Medical Oncology, ${ }^{4}$ Zhejiang Key Laboratory of \\ Diagnosis \& Treatment Technology on Thoracic Oncology (Lung and Esophagus), Institute of Cancer Research and Basic Medical Sciences of \\ Chinese Academy of Sciences, Cancer Hospital of University of Chinese Academy of Sciences, Zhejiang Cancer Hospital, Hangzhou 310022 , China \\ Contributions: (I) Conception and design: H Lu; (II) Administrative support: H Lu; (III) Provision of study materials or patients: C Li; (IV) \\ Collection and assembly of data: L Xu, C Li; (V) Data analysis and interpretation: L Xu; (VI) Manuscript writing: All authors; (VII) Final approval of \\ manuscript: All authors. \\ \#These authors contributed equally to this work. \\ Correspondence to: Hongyang Lu. Zhejiang Key Laboratory of Diagnosis \& Treatment Technology on Thoracic Oncology (Lung and Esophagus), \\ Institute of Cancer Research and Basic Medical Sciences of Chinese Academy of Sciences, Cancer Hospital of University of Chinese Academy of \\ Sciences, Zhejiang Cancer Hospital, Hangzhou 310022, China. Email: luhy@zjcc.org.cn.
}

\begin{abstract}
Invasive mucinous adenocarcinoma (IMA) is a unique histological subtype of adenocarcinoma. Due to its low incidence rates, survival data for IMA is scarce and often contradictory. The clinical manifestations of IMA are not precise as compared to other adenocarcinomas, with some patients having bronchial mucus overflow. Difference in immunohistochemical expression levels is present in IMA and invasive non-mucinous adenocarcinomas (INMA). Kirsten rat sarcoma viral oncogene homolog (KRAS) mutations are more frequent in IMAs, while epidermal growth factor receptor (EGFR) mutations are relatively rare. This makes it distinct from the other more common adenocarcinomas. Neuregulin 1 (NRG1) gene fusions are considered important therapeutic targets for IMA, suggesting that Afatinib may be an effective drug to treat IMA. However, IMA prognosis remains controversial.
\end{abstract}

Keywords: Invasive mucinous adenocarcinoma (IMA); clinicopathologic features; kirsten rat sarcoma viral oncogene homolog (KRAS); neuregulin-1 (NRG1); afatinib; prognosis

Submitted Jul 28, 2019. Accepted for publication Oct 17, 2019.

doi: $10.21037 /$ tcr.2019.11.02

View this article at: http://dx.doi.org/10.21037/tcr.2019.11.02

\section{Introduction}

Lung cancer is the leading cause of cancer-related deaths worldwide (1). Invasive mucinous adenocarcinoma (IMA) is a subtype of lung adenocarcinoma, and can be classified as acinar adenocarcinoma, papillary adenocarcinoma, bronchioloalveolar carcinoma (BAC) or solid adenocarcinoma with mucus formation. However, the World Health Organization (WHO, 1981) has not classified IMA to-date (2). Kish et al. (3) first proposed the concept of pulmonary mucinous adenocarcinoma in a case report published in 1989. Since then, the definition of lung mucinous adenocarcinoma has been revised over the decades $(4,5)$. WHO updated their classification of lung cancer in 2015, with lung mucinous adenocarcinoma being divided into mucinous adenocarcinoma in situ (AIS), microinvasive mucinous adenocarcinoma, IMA and colloidal adenocarcinoma. Of these, IMA is the most common. IMA was formerly classified as mucinous BAC, where tumor cells had a goblet or columnar morphology and abundant intracytoplasmic mucin (6). IMA has a low incidence rate, a lack of specific clinical manifestations and unclear prognosis. With advancements in genetic screening technologies, IMA has been associated with mutations in kirsten rat sarcoma viral oncogene homolog (KRAS), anaplastic lymphoma kinase (ALK) rearrangements and neuregulin-1 (NRG1) fusions. Associated gene mutations and signaling pathways may be attractive targets for the treatment of IMA. The 
frequency of epidermal growth factor receptor (EGFR) mutations in IMA are lower compared to other subtypes of adenocarcinomas $(7,8)$. Afatinib is a second-generation EGFR tyrosine kinase inhibitor (EGFR-TKI) that has shown good efficacy in patients with EGFR mutationpositive lung adenocarcinomas. Interestingly, Afatinib had an obvious beneficial effect in a few patients with NRG1 fusions. This review will focus on the clinicopathological, molecular, treatment strategies and prognosis of IMA.

\section{Clinicopathological features of IMA}

IMA is a unique histologic subtype of lung adenocarcinoma, and may manifest with non-mucinous adenocarcinomas (NMA) (6). When IMA is of the mixed type, i.e., with non-mucinous components, adherent growth, acinar, papillary and micropapillary carcinoma, and where the NMA components makes up $\geq 10 \%$ of the tumor, diagnosis should be invasive mucinous carcinoma and non-mucinous mixed adenocarcinoma. In addition to the diagnosis, NMA components should be indicated (6). The characteristic pathological manifestations of IMA consist of the presence of goblet or columnar tumor cells with abundant intracytoplasmic mucin, with basally localized nuclei that is rarely observed in other lung adenocarcinomas (6). Multiple growth patterns such as acinar, papillary, micropapillary, or lepidic can be observed in IMAs. The lepidic growth pattern is the most common that is observed.

IMA accounts for approximately $2-10 \%$ of lung adenocarcinomas (8-10). The epidemiology of IMA is similar to that of other lung adenocarcinomas as reported by WHO (6). Moon et al. (11) reviewed primary lung NMA and IMA from 2000 to 2014 extracted from the Surveillance, Epidemiology, and End Results (SEER) database. They found that the proportion of female IMA patients was higher (IMA: male to female ratio 1:1.45; NMA: 1:1.09). The average age at the time of diagnosis for IMA was similar to NMA (65.97 vs. 66.09 years old). In addition, the study found that the proportion of whites, blacks and other ethnic groups was $80 \%, 17.1 \%$ and $8.1 \%$, respectively. IMAs were found to be localized in the lower lobe and were well differentiated. With regards to staging, IMA tends to be $\mathrm{N} 0$ (91.7\% vs. $72.3 \%, \mathrm{P}<0.001)$, T1 or T2 $(\mathrm{P}<0.001)$, and stage I tumors $(\mathrm{P}<0.001)$ compared to NMAs. There was no significant correlation between smoking and IMA, however, 10 of the 13 IMA patients reported by Yoshizawa et al. (9) and 15 of the 25 IMA patients reported by Duruisseaux et al. (12) had a history of smoking. Smoking may contribute to the development and prognosis of IMA. Boland et al. (13) reported that the overall survival $(\mathrm{OS})$ rate $(\mathrm{P}=0.006)$ and progression-free survival $(\mathrm{PFS})$ rate $(\mathrm{P}=0.024)$ were worse in smokers with mucinous tumors with scaly abundance after multivariate analysis. The correlation between smoking and IMA needs to be investigated using larger patient cohorts.

IMA displays a strong tendency for multicentric, multilobar and bilateral lung involvement and appears to develop well in the lower lobes of the lungs (14). It is often observed with segmental or lobular consolidation with multi-lobal solid or subsolid nodules or masses, and bronchial aeration (5). However, large, thin-walled cysts are rare. Masuzawa et al. (15) reported a 75-yearold male who underwent a bronchoscopy biopsy. IMA was diagnosed upon histopathology confirmation, with imaging demonstrating irregular large cystic lesions. IMA lesions during the early stages are easily misdiagnosed as lobar pneumonia, tuberculosis or other diffuse lung lesions. Clinical definitive diagnosis of malignancy via biopsies is often challenging. This is because the majority of IMAs are located at the edges of the lower lung lobe, and the alveolar cavity surrounding the tumor is usually filled with mucin. Hence bronchoscopy biopsies are usually inadequate. Percutaneous computed tomography (CT)-guided lung biopsies may be more helpful for a diagnosis, however surgical resection pathological diagnosis of gross specimens is still the most effective method.

Differences in immunohistochemical expression levels are present in IMA and INMA. IMAs typically express cytokeratin7 (CK7) (88-94.7\%) and cytokeratin 20 (CK20) ( 79\%) (16-19). The expression levels of thyroid transcription factor-1 (TTF-1) (11-27.5\%) and noval aspartic proteinase of the pepsin family A (Napsin A) $(11 \%)$ are lower in IMA compared to INMA. TTF-1 is expressed in about $75-80 \%$ of adenocarcinomas, especially in terminal respiratory unit (TRU) morphology $(19,20)$. Yatabe et al. (21) reported $88 \%$ of adenocarcinomas with TRU morphology expressed TTF-1. IMAs are generally considered to be non-TRU types. Non-TRU type tumors are associated with a worse prognosis compared to TRU type tumors (22). Goldstein NS and Thomas MA compared the expression levels of TTF, CK7, CK20 and villin in 14 mucinous and 26 non-mucinous BACs and found that 3 of the 14 mucinous neoplasms had weak TTF reactivity (16). In contrast, 24 of 26 non-mucinonus BACs were strongly TTF reactive. Eleven of the mucinous BACs had CK20 reactivity, whereas only one non-mucinous BAC had 
Table 1 Current target and therapy of IMA

\begin{tabular}{llll}
\hline Target & Incidence rate & Drug & Therapeutic effect \\
\hline EGFR & $0-5 \%(24-27)$ & EGFR TKI inhibitors & Effective, need more clinical data \\
ALK & $2.2 \%(8)$ & ALK inhibitors & Effective, need more clinical data \\
NRG1 & $7-27 \%(12,25,28)$ & Afatinib $(29,30)$ & Case reports shows effective \\
KRAS & $63-90 \%(6,8,24,31)$ & Sterminib (AZD6244) with BYL719 (32) & Effective in vivo \\
B7-H3 & $42.4 \%(33)$ & Unclear & Need further study \\
PD-L1 & $0-6.1 \%(33-35)$ & Immune checkpoint inhibitors & Need more clinical data \\
\hline
\end{tabular}

IMA, invasive mucinous adenocarcinoma; EGFR, epidermal growth factor receptor; ALK, anaplastic lymphoma kinase; NRG1, neuregulin-1; KRAS, kirsten rat sarcoma viral oncogene homolog.

reactivity. In addition, one mucinous $B A C$ had villin reactivity. Mucinous BACs are usually TTF and villin nonreactive and CK20 reactive. All the mucinous and nonmucinous BACs had strong CK7 reactivity in more than $50 \%$ of the neoplastic cells. However, the evaluation of TTF-1 and CK20 expression may have limited diagnostic value in the context of identifying mucinous BACs and extrapulmonary mucinous tumor metastasis in the lungs (17). Wu et al. (19) found that napsin A immunoreactivity was observed in $2(11 \%)$ of 18 IMA patients. Kunii et al. (23) reported hepatocyte nuclear factor $4 \alpha(\mathrm{HNF} 4 \alpha)$ expression in thyroid TTF-1-negative lung adenocarcinomas. A total of 262 patients with pulmonary adenocarcinoma were examined both histologically and immunohistochemically. TTF-1 was expressed in 222 of the patients, with 40 patients being negative. Of the 40 TTF-1-negative patients, there were 31 patients with mucinous-type tumors, and $34(85 \%)$ patients with tumors expressing HNF4 $\alpha$. Mucin 5ac (MUC5AC) and mucin 2 (MUC2) were also expressed in TTF-1 negative tumors. TTF-1 expression had a significant inverse correlation with $\mathrm{HNF} 4 \alpha$ and MUC5AC expression. Similar results were observed in Tsuta et al. (18). MUC5, MUC6 and CK7 were highly expressed in 40 mucinous BACs patients, i.e., $97.5 \%$, $75 \%$ and $94.7 \%$ respectively, while MUC1 was poorly expressed (57.5\%).

\section{Molecular features of IMA}

IMA has distinct molecular characteristics (Table 1). Recent studies have demonstrated multiple genetic abnormalities in IMA. High throughput analyses have revealed several genetic abnormalities in IMA including mutations in KRAS, $\mathrm{v}$-raf murine sarcoma viral oncogene homolog B1 (BRAF), v-erb-b2 erythroblastic leukemia viral oncogene homolog
2 (ERBB2) and phosphatidylinositol-3 kinase catalytic alpha (PIK3CA), while NRG1, neurotrophic tyrosine kinase receptor type 1(NTRK1), ALK, rearranged during transfection (RET) and v-erb-a erythroblastic leukemia viral oncogene homolog 4 (ERBB4) rearrangements, and tumor protein 53 (TP53) mutations were rare $(24,25)$. ALK rearrangements were mutually exclusive with KRAS mutations, while simultaneous ALK rearrangements with EGFR mutations were common (36).

KRAS mutations are the most frequent oncogenic driver mutations in IMAs ( $63 \%$ to $90 \%$ ), which is often observed with other mutations. However, other clinical and genetic features are not clearly established. The most common mutations are G12D and G12V in IMAs and G12C in INMAs $(6,8,24,31)$. Boland et al. (13) investigated 760 patients with lung adenocarcinomas and identified 57 cases of IMAs and 54 cases of mixed mucinous adenocarcinomas. KRAS mutations were common $(76 \%$ of IMAs, $68 \%$ of mixed mucinous/non-mucinous), with $38 \%$ of KRAS mutations occurring with other mutations, especially STK11. Three patients with ALK, two patients with EGFR and one patient with BRAF V600E mutations were identified. Interestingly, all ALK rearrangements were present in mixed mucinous/non-mucinous tumors. The study performed by Tsuta et al. (8) investigated 904 patients with postoperative lung adenocarcinomas. Retrospective analysis demonstrated that KRAS mutations were more frequent in IMA patients compared to other subtypes of lung adenocarcinoma and were observed in 33 of 45 IMA patients. A retrospective analysis of 864 patients with lung adenocarcinoma after surgery performed by Kadota et al. (37) showed that the incidence of KRAS mutations in patients with simple IMA was significantly higher compared to mixed IMA. KRAS point mutations (G-A) 
are more frequently observed in IMA patients compared to G-T or G-C substitutions. It has been demonstrated that extracellular mucin and mucin histological patterns in lung adenocarcinomas are closely associated with KRAS mutations. Meng et al. (38) analyzed KRAS mutations and survival rate in 6,939 NSCLC patients and found that OS in patients with early and mid-stage lung adenocarcinoma with KRAS mutations were significantly lower. Hence, KRAS mutations may also be associated with poor IMA prognosis.

ALK rearrangements have also been observed in IMA patients. Tsuta et al. (8) reported that ALK rearrangements were more prevalent in micropapillary-predominant adenocarcinomas (MPAs) (15.0\%), while the frequency of ALK rearrangements in IMA patients was $2.2 \%$. Qu et al. (39) detected ALK rearrangements in 25 of the 73 pulmonary mucinous adenocarcinoma tumor samples. However, their study included solid-based tumors with mucin-producing adenocarcinomas and lung signet ring cell carcinomas, which are not considered IMAs in the current classification.

Several trials have shown that NMA has a high EGFR mutation rate compared to IMA (only 0-5\%) (24-27). Wang et al. (40) evaluated the clinicopathological variables and EGFR mutation status of 376 patients with lung adenocarcinoma. They found that acinar was the predominant histological subtype (39.9\%), followed by papillary (17.8\%), solid (14.9\%), lepidic $(10.1 \%)$, AIS $(5.8 \%)$, micropapillary $(5.1 \%)$, minimally invasive adenocarcinoma (MIA) (4.0\%), and IMA (2\%). In a multicenter prospective phase II trial (IFCT0401) performed by Wislez et al. (41), 50 samples were obtained and subdivided into: non-mucinous $(\mathrm{n}=25)$ and mucinous $(\mathrm{n}=25)$ subtypes. No EGFR mutations in exons 18-21 were detected in the mucinous group, and no objective response was observed in patients with mucinous adenocarcinoma treated with gefitinib.

Gene fusions in NRG1 are considered novel and promising molecular targets for IMA. The estimated frequencies of these fusions are approximately $7-27 \%(12,25,28)$. NRG1 is a ligand protein and a member of the growth factor family and are structurally related to epidermal growth factor (EGF) and are responsible for activating ErbB receptor tyrosine kinases (29). NRG1 fusion leads to continual activation of the human epidermal growth factor receptor-2 (HER2) and human epidermal growth factor receptor-4 (HER4) signaling pathways. This leads to uncontrolled cell proliferation and tumorigenesis. NRG1 has important functions in the nervous system, heart and breast. In addition, NRG signaling is closely associated with the development and function of several organ systems and human diseases (42). Preclinical studies have suggested that increased expression and activation of neuroproteins may trigger cancer (43). NRG1 is usually not expressed in normal lung tissue and lung adenocarcinomas, however NRG1 III-b3 fusions are observed in IMA. Similar to ERBB2-ERBB3 dimers, phoshoinisitide-3-kinase (PI3K)/AKT and mitogen-activated protein kinase (MAPK) pathways could be targetable. NRG1 fusions too could be promising therapeutic targets (44). Several NRG1 gene fusions observed in IMA patients include CD74 (the most common), SLC3A2, VAMP2, RBPMS and SDC4 $(25,45,46)$. Fernandez-Cuesta et al. (28) transduced H322 and H1568 lung cancer cell lines that express normal levels of ERBB2 and ERBB3 with retrovirus expressing CD74-NRG1 fusion transcripts. They found that the levels of p-ERBB2, p-ERBB3, p-AKT, and p-S6K were significantly increased in $\mathrm{H} 322$ and H1568 cells expressing CD74-NRG1 compared to empty vector controls and cells expressing CD74-NRG1 $\triangle \mathrm{EGF}$ (a truncated version of the fusion lacking the EGF-like domain). In addition, a strong association between the EGF-like domain and increased p-ERBB3 and p-AKT levels were observed. This study confirmed that CD74-NRG1 was the driver gene through which overexpression of the EGF like domain of NRG1 III- $\beta 3$ induced phosphorylation of ERBB 3 to subsequently activate the downstream PI3K-AKT pathway. The oncogenic properties of SLC3A2-NRG1 and VAMP2-NRG1 fusions were observed to be similar $(46,47)$. Fernandez-Cuesta et al. (28) performed transcriptome sequencing on 25 nonsmokers who had KRAS and EGFR gene-negative lung adenocarcinomas. Fifteen of the patients were diagnosed with IMA and were negative for mutations in the following genes; KRAS, EGFR, ALK, ROS1, RET, BRAF and Her2. Four of the 15 patients (27\%) were positive for CD74NRG1 fusions as measured by RT-PCR. Interestingly, the four patients who had CD74-NRG1 fusions were diagnosed with IMA and were Asian. This suggests that CD74-NRG1 fusions appear to be mutually exclusive to known oncogenic mutations, including EGFR/KRAS/BRAF/ERBB2/ALK/ ROS1. In addition, patients were all non-smokers and Asians. The authors also demonstrated that CD74-NRG1 signaled through ERBB2-ERBB3 heterodimers to activate the PI3K/ AKT pathway to induce oncogenic growth. Hence, CD74NRG1 fusions may represent a therapeutic opportunity for IMA. This is based on several available drugs that target ERBB2, ERBB3, and their downstream pathways (48). Shin et al. (47) reported that in 59 patients with IMA, NRG1 
Table 2 Case reports of afatinib on NRG1-positive advanced NSCLC

\begin{tabular}{|c|c|c|c|c|c|c|c|c|c|}
\hline $\begin{array}{l}\text { Author (afatinib, } \\
40 \text { mg qd) }\end{array}$ & $\begin{array}{l}\text { The pathologic } \\
\text { types }\end{array}$ & Case(s) & $\begin{array}{l}\text { Smoking } \\
\text { history }\end{array}$ & Sex & Race & Age & Fusion type & PFS & $\begin{array}{l}\text { The best } \\
\text { response }\end{array}$ \\
\hline Gay et al. (29) & Adenocarcinoma & 2 & Nonsmoker & Male & Caucasian & 42-year-old & SLC3A2-NRG1 & 12 months & Partial response \\
\hline Cheema et al. (30) & IMA & 1 & Nonsmoker & Female & Asian & 62-year-old & CD74-NRG1 & 26 weeks & Partial response \\
\hline
\end{tabular}

NRG1, neuregulin-1.

fusions were observed in 16 patients, with NRG1-SLC3A2 fusions accounting for $81.25 \%$ (13 patients), and CD74NRG1 accounting for $18.75 \%$ (3 patients). In addition, 10 NRG1 fusions with KRAS mutations (62.5\%) and 2 NRG1 fusions with NRAS Q61L mutations and EML4-ALK fusions were also observed. Survival analysis indicated that OS for IMA patients with NRG1-fusions was significantly worse compared to patients without NRG1 gene fusions. The study also found that SLC3A2-NRG1 fusions increased phosphorylation and heteroduplex formation of ERBB2ERBB3 and activated the downstream PI3K/AKT/mTOR pathway via paracrine signaling. This ectopic expression in lung cancer cells increases the possibility of tumor cell growth and proliferation, suggesting that SLC3A2-NRG1 fusions are a driving factor for IMA, and hence for poor prognosis. In summary, NRG1 is an important driver oncogene for IMA and may be an important therapeutic target.

\section{Treatment for IMA}

As a subtype of lung adenocarcinoma, IMA is treated the same way as other adenocarcinomas. Surgery is the preferred treatment option for IMA patients, except for patients with stage IIIB and IV (49). Lobectomy with lymphadenectomy is considered the standard treatment, while sublobar resection could be performed for early-stage lung cancers or for patients with low pulmonary function. In a large cohort retrospective study, Moon et al. (11) found that patients with primary M0 lung adenocarcinomas, the histological type of the tumor did not affect survival time and was independent of IMA status. Hence early stage IMA patients can still benefit from surgery, and early diagnosis is essential. Platinum-based post-surgical adjuvant chemotherapy remains the standard treatment for II-IIIA NSCLC (50). Whether there is a significant difference in curative rates remains to be determined in larger cohort studies. Patients receiving non-TKI platinum-based conventional chemotherapy (CTx) with stage IV IMA did not have a significant change on OS compared to untreated IMA patients (51). Hence, new therapeutic approaches are required for IMAs of the lung.

EGFR-TKIs could be used as first-line therapy for advanced EGFR-mutant NSCLC (52). The data for efficacy of EGFR-TKIs in patients with IMA is limited. KRAS mutations are frequently observed in IMA patients. Hence, several studies have suggested that mutant KRAS could be targeted for therapeutic intervention. Ku et al. (32) suggested that sterminib (AZD6244) combined with BYL719 could improve the treatment efficacy for KRAS mutant NSCLC. However, it has not been used in clinical practice to-date. ALK inhibitors have been demonstrated to be effective in NSCLC patients with ALK rearrangements, hence it's efficacy for IMA patients harboring ALK rearrangements is worth being investigated.

As the second EGFR-TKI developed, afatinib is an irreversible oral formulation of ErbB family of inhibitors that selectively block EGFR, HER2 and HER4. Afatinib has demonstrated a significant survival benefit over platinumbased chemotherapy as a first-line treatment for patients with advanced NSCLC harboring EGFR mutations (29). Multiple studies have shown that afatinib is effective in lung adenocarcinoma patients with NRG1 fusions $(29,30,53)$ (Table 2). Gay et al. (29) reported on two lung cancer patients with NRG1 fusions treated with afatinib. Their PFS time was 12 and 10 months. Cheema et al. (30) also reported a 62-year-old Asian woman who never smoked having multiple lung tumors with no distant metastases. Pulmonary mass punctures confirmed the pathological type as IMA but with no common gene mutations, such as EGFR and ALK. Gene sequencing demonstrated that the patient had a CD74-NRG1 fusion. The patient was treated with afatinib (40 $\mathrm{mg} \mathrm{qd}$ ) and imaging results showed a partial response that was maintained for 26 weeks. Larger cohort studies are needed to confirm the benefit of afatinib 
for the treatment of patients with NRG1 fusions.

Immune checkpoint blockade therapies have shown promise for the treatment of malignant tumors in recent years. Key immunological checkpoint proteins include programmed death 1 (PD-1) and its ligand PD-L1. Miyazawa et al. (34) sampled 90 NSCLC tumor tissues encompassing various adenocarcinoma subtypes. Out of the 14 acinar predominant adenocarcinomas, only 8 were PD-L1 positive (defined as TPS $\geq 1 \%$ ), 6 out of 11 solid predominant adenocarcinomas were PD-L1 positive and 0 out of 4 IMAs were PD-L1 positive. Larger patient numbers are needed to substantiate these findings. Similarly, Shimizu et al. (35) detected very low PD-L1 expression in 25 IMA. Nakagomi et al. (33) reported that PD-L1 expression in IMA and conventional adenocarcinomas were $6.1 \%$ and $59.7 \%$, respectively, while $\mathrm{B} 7-\mathrm{H} 3$ expression in IMA was as high as $42.4 \%$. Hence $\mathrm{B} 7-\mathrm{H} 3$ maybe a potential immunotherapeutic target.

\section{Prognosis of IMA}

Due to the low incidence rates, patient survival data for IMA is limited and often contradictory. Retrospective studies by Yoshizawa et al. (9) performed Kaplan-Meier and Cox regression analysis on 514 patients with stage I lesions. Based on disease-free survival (DFS), three overall prognostic groups with low, medium, and high-grade clinical behaviors were identified. Low grade included in situ adenocarcinoma (1 patient) and MIA (8 patients), both of which were $100 \%$ DFS. Mid-grade tumors were non-mucinous lepidic predominant (29 patients, 5-year DFS 90\%), acinar predominance (232 patients, 5-year DFS $84 \%$ ), papillary predominant adenocarcinoma (143 patients, 5 -year DFS $83 \%)$. High-grade tumors included: solid predominant (67 patients, 5 -year DFS 70\%), micropapillary predominant (12 patients, 5-year DFS 67\%), colloid predominant (9 patients, 5-year DFS 71\%) and invasive mucinous and mixed mucinous/non-mucinous (13 patients, 5-year DFS 76\%). Similarly, Russell et al. (31) suggested that IMA was a subtype of lung adenocarcinoma with poor prognosis. Interestingly, Warth et al. (10) reached the contradictory conclusion in their retrospective analysis of 500 adenocarcinomas patients (stages I to IV). IMA ( $\mathrm{n}=12$ ) compared to 487 conventional adenocarcinomas had better OS (88.7 vs. 63.5 months), disease-specific survival (DSS) (all survived vs. 73.3 months), and DFS (88.1 vs. 56.1 months). In addition, Shim et al. (24) in a retrospective study of patients in the United States and South Korea showed no statistically significant differences in 5-year survival and OS between IMA and NMA patients, with all IMA recurrences being intrapulmonary. However, the IMA patient cohort was small, with only 72 patients. Interestingly, different phenotypes of IMA also influence prognosis. Lin et al. (54) reported on the prognosis of IMA with different growth patterns. Patients were identified as having lepidicpredominant IMA and 10 as having acinar-predominant IMA. The proportion of lymph node metastasis were significantly higher in the acinar-predominant IMA $(\mathrm{P}=0.046)$. Survival analysis showed that acinar-predominant IMA had a poorer prognosis $(\mathrm{P}=0.0294)$. In addition, Shimizu et al. (35) observed that the pneumonic type was correlated with a significantly worse outcome in IMA and a higher rate of recurrence. In summary, the clinical features of lung IMA, especially recurrence characteristics and prognosis, deserve further investigation.

\section{Conclusions}

In summary, IMA is a subtype of lung adenocarcinoma, of which the epidemiology is similar to other lung adenocarcinomas. However, imaging techniques and pathology could be used to differentiate IMA. Due to its low incidence rates, survival data for IMA patients is scarce and often contradictory. KRAS mutations frequently occur in IMAs while EGFR mutations are rare. PD-L1 expression in IMA patient tumors is very low (Table 1). Patients with IMA at the early stages can benefit from surgery and postoperative chemotherapy. Efficacy data for EGFR-TKI in IMA patients with advanced EGFR mutations is lacking and needs further study. NRG1 fusions are considered to be an important driver gene for IMA. Afatinib has good efficacy for the treatment of lung cancer patients with NRG1-fusions, however larger prospective cohort studies are needed to confirm these observations.

\section{Acknowledgments}

Funding: This work was supported by the Zhejiang Province Medical Science Project of China (No.2019ZH019), Wu Jieping Medical Foundation (320.6750.19050) and the 1022 Talent Training Program of Zhejiang Cancer Hospital.

\section{Footnote}

Conflicts of Interest: All authors have completed the ICMJE uniform disclosure form (available at http://dx.doi. 
org/10.21037/tcr.2019.11.02). The authors have no conflicts of interest to declare.

Ethical Statement: The authors are accountable for all aspects of the work in ensuring that questions related to the accuracy or integrity of any part of the work are appropriately investigated and resolved.

Open Access Statement: This is an Open Access article distributed in accordance with the Creative Commons Attribution-NonCommercial-NoDerivs 4.0 International License (CC BY-NC-ND 4.0), which permits the noncommercial replication and distribution of the article with the strict proviso that no changes or edits are made and the original work is properly cited (including links to both the formal publication through the relevant DOI and the license). See: https://creativecommons.org/licenses/by-nc-nd/4.0/.

\section{References}

1. Siegel RL, Miller KD, Jemal A. Cancer statistics. CA Cancer J Clin 2015;65:5-29.

2. WHO. Histological typing of lung tumors. Am J Clin Pathol 1982;77:123-36.

3. Kish JK, Ro JY, Ayala AG, et al. Primary mucinous adenocarcinoma of the lung with signet-ring cells: a histochemical comparison with signet-ring cell carcinomas of other sites. Hum Pathol 1989;20:1097-102.

4. Travis WD, Brambilla E, Müller-Hermelink HK, et al. World Health Organization classification of tumours: pathology and genetics of tumors of the lung, pleura, thymus and heart. Lyon: IARC Press, 2004:9-124.

5. Travis WD, Brambilla E, Noguchi M, et al. International association for the study of lung cancer/American thoracic society/European respiratory society international multidisciplinary classification of lung adenocarcinoma. J Thorac Oncol 2011;6:244-85.

6. Travis WD, Brambilla E, Burke AP et al. WHO Classification of Tumours of the Lung, Pleura, Thymus and Heart. Lyon: International Agency for Research on Cancer 2015.

7. Cha YJ, Shim HS. Biology of invasive mucinos adenocarcinoma of the lung. Transl Lung Cancer Res 2017;6: 508-12.

8. Tsuta K, Kawago M, Inoue E, et al. The utility of the proposed IASLC/ATS/ERS lung adenocarcinoma subtypes for disease prognosis and correlation of driver gene alterations. Lung Cancer 2013;81:371-6.
9. Yoshizawa A, Motoi N, Riely GJ, et al. Impact of proposed IASLC/ATS/ERS classification of lung adenocarcinoma: prognostic subgroups and implications for further revision of staging based on analysis of 514 stage I cases. Mod Pathol 2011;24:653-64.

10. Warth A, Muley T, Meister M, et al. The novel histologic International Association for the Study of Lung Cancer/ American Thoracic Society/European Respiratory Society classification system of lung adenocarcinoma is a stageindependent predictor of survival. J Clin Oncol 2012;30: 1438-46.

11. Moon SW, Choi SY, Moon MH. Effect of invasive mucinous adenocarcinoma on lung cancer-specific survival after surgical resection: a population-based study. J Thorac Dis 2018;10:3595-608.

12. Duruisseaux M, Mcleer-Florin A, Antoine M, et al. NRG1 fusion in a French cohort of invasive mucinous lung adenocarcinoma. Cancer Med 2016;5:3579-85.

13. Boland JM, Maleszewski JJ, Wampfler JA, et al. Pulmonary invasive mucinous adenocarcinoma and mixed invasive mucinous/nonmucinous adenocarcinomaa clinicopathological and molecular genetic study with survival analysis. Human Pathology 2018;71:8-19.

14. Simsir A, Wei XJ, Yee H, et al. Differential expression of cytokeratins 7 and 20 and thyroid transcription factor- 1 in bronchioloalveolar carcinoma: an immunohistochemical study in bronchioloalveolar carcinoma: an immunohistochemical study in fine-needle aspiration biopsy specimens. Am J Clin Pathol 2004;121:350-7.

15. Masuzawa K, Minematsu N, Sasaki M, et al. Invasive mucinous adenocarcinoma of the lung presenting as a large, thin-walled cyst: A case report and literature review. Mol Clin Oncol 2017;6:433-7.

16. Goldstein NS, Thomas M. Mucinous and nonmucinous bronchioloalveolar adenocarcinomas have distinct staining patterns with thyroid transcription factor and cytokeratin 20 antibodies. Am J Clin Pathol 2001;116:319-25.

17. Lau SK, Desrochers MJ, Luthringer DJ. Expression of thyroid transcription factor-1, cytokeratin 7 , and cytokeratin 20 in bronchioloalveolar carcinomas: an immunohistochemical evaluation of 67 cases. Mod Pathol 2002;15:538-42.

18. Tsuta K, Ishii G, Nitadori J, et al. Comparison of the immunophenotypes of signet-ring cell carcinoma, solid adenocarcinoma with mucin production, and mucinous bronchioloalveolar carcinoma of the lung characterized by the presence of cytoplasmic mucin. J Pathol 2006;209:78-87. 
19. Wu J, Chu PG, Jiang Z, et al. Napsin A expression in primary mucin-producing adenocarcinomas of the lung: an immunohistochemical study. Am J Clin Pathol 2013;139:160-6.

20. Krasinskas AM, Chiosea SI, Pal T, et al. KRAS mutational analysis and immunohistochemical studies can help distinguish pancreatic metastases from primary lung adenocarcinomas. Mod Pathol 2014;27:262-70.

21. Yatabe Y, Mitsudomi T, Takahashi T. TTF-1 expression in pulmonary adenocarcinomas. Am J Surg Pathol 2002;26:767-73.

22. Sumiyoshi S, Yoshizawa A, Sonobe M, et al. Non-terminal respiratory unit type lung adenocarcinoma has three distinct subtypes and is associated with poor prognosis. Lung Cancer 2014;84:281-8.

23. Kunii R, Jiang S, Hasegawa G, et al. The predominant expression of hepatocyte nuclear factor 4alpha (HNF4alpha) in thyroid transcription factor-1 (TTF-1)negative pulmonary adenocarcinoma. Histopathology 2011;58:467-76.

24. Shim HS, Kenudson M, Zheng Z, et al. Unique Genetic and Survival Characteristics of Invasive Mucinous Adenocarcinoma of the Lung. J Thorac Oncol 2015;10:1156-62.

25. Nakaoku T, Tsuta K, Ichikawa H, et al. Druggable oncogene fusions in invasive mucinous lung adenocarcinoma. Clin Cancer Res 2014;20:3087-93.

26. Hwang DH, Sholl LM, Rojas-Rudilla V, et al. KRAS and NKX2-1 Mutations in Invasive Mucinous Adenocarcinoma of the Lung. J Thorac Oncol 2016;11:496-503.

27. Finberg KE, Sequist LV, Joshi VA, et al. Mucinous differentiation correlates with absence of EGFR mutation and presence of KRAS mutation in lung adenocarcinomas with bronchioloalveolar features. J Mol Diagn 2007;9:320-6.

28. Fernandez-Cuesta L, Plenker D, Osada H, et al. CD74NRG1 fusions in lung adenocarcinoma. Cancer Discov 2014;4:415-22.

29. Gay ND, Wang Y, Beadling C, et al. Durable response to Afatinib in lung adenocarcinoma harboring NRG1 gene fusions. J Thorac Oncol 2017;12:e107-10.

30. Cheema PK, Doherty M, Tsao MS. A case of invasive mucinous pulmonary adenocarcinoma with a CD74NRG1 fusion protein targeted with Afatinib. J Thorac Oncol 2017;12:e200-2.

31. Russell PA, Wainer Z, Wright GM, et al. Does lung adenocarcinoma subtype predict patient survival? A clinicopathologic study based on the new International
Association for the Study of Lung Cancer/American Thoracic Society/European Respiratory Society international multidisciplinary lung adenocarcinoma classification. J Thorac Oncol 2011;6:1496-504.

32. Ku BM, Jho EH, Bae YH, et al. BYL719, A selective inhibitors of phosphoinositide 3 -Kinase $\alpha$, enhances the effect of selumetinib (AZD6244, ARRY-142886) in KRASMutant non -small cell lung cancer. Invest New Drugs 2015;33:12-21.

33. Nakagomi T, Goto T, Hirotsu Y, et al. Genomic Characteristics of Invasive Mucinous Adenocarcinomas of the Lung and Potential Therapeutic Targets of B7-H3. Cancers 2018;10:478.

34. Miyazawa T, Marushima H, Saji H, et al. PD-L1 Expression in Non-Small-Cell Lung Cancer Including Various Adenocarcinoma Subtypes. Ann Thorac Cardiovasc Surg 2019;25:1-9.

35. Shimizu K, Okita R, Saisho S, et al. Clinicopathological and immunohistochemical features of lung invasive mucinous adenocarcinoma based on computed tomography findings. Onco Targets Ther 2016;10:153-63.

36. Wang J, Dong Y, Cai Y, et al. Clinicopathologic characteristics of ALK rearrangements in primary lung adenocarcinoma with identified EGFR and KRAS status. J Cancer Res Clin Oncol 2014;140:453-60.

37. Kadota K, Yeh YC, D'Angelo SP, et al. Associations between mutations and histologic patterns of mucin in lung adenocarcinoma: invasive mucinous pattern and extracellular mucin are associated with KRAS mutation. Am J Surg Pathol 2014;38:1118-27.

38. Meng D, Yuan M, Li X, et al. Prognostic value of K-RAS Mutations in patients with non -small cell lung cancer: a Systematic review with meta -analysis. Lung Cancer 2013;81:1-10.

39. Qu Y, Che N, Zhao D, et al. The clinicopathological significance of ALK rearrangements and KRAS and EGFR mutations in primary pulmonary mucinous adenocarcinoma. Tumour Biol 2015;36:6417-24.

40. Wang T, Zhang Y, Liu B, et al. Associations between epidermal growth factor receptor mutations and histological subtypes of lung adenocarcinoma according to the IASLC/ATS/ERS classification in Chinese patients. Thoracic Cancer 2017;8:600-5.

41. Wislez M, Antoine M, Baudrin L, et al. Non-mucinous And mucinous subtypes of adenocarcinoma with bronchioloalveolar carcinoma features differ by biomarker expression and in the response to gefitinib. Lung Cancer 2010;68:185-91. 
42. Falls DL. Neuregulins: functions, forms, and signaling strategies. Exp Cell Res 2003;284:14-30.

43. Montero JC, Rodríguez-Barrueco R, Ocaña A, et al. Neuregulins and cancer. Clin Cancer Res 2008;14:3237-41.

44. Fernandez-Cuesta L, Thomas RK. Molecular pathways: targeting NRG1 fusions in lung cancer. Clin Cancer Res 2015;21:1989-94.

45. Dhanasekaran SM, Balbin OA, Chen G, et al.

Transcriptome meta-analysis of lung cancer reveals recurrent aberrations in NRG1 and Hippo pathway genes. Nat Commun 2014;5:5893.

46. Jung Y, Yong S, Kim P, et al. VAMP2-NRG1 fusion gene is a novel oncogenic driver of non-small-cell lung adenocarcinoma. J Thorac Oncol 2015;10:1107-11.

47. Shin DH, Lee D, Hong DW, et al. Oncogenic function and clinical implications of SLC3A2 - NRG1 fusion in invasive mucinous adenocarcinoma of the lung. Oncotarget 2016;7:69450-65.

48. Yarden Y, Pines G. The ERBB network: at last, cancer therapy meets systems biology. Nat Rev Cancer 2012;12:553-63.

49. Schneider BJ, Daly ME, Kennedy EB, et al. Stereotactic body radiotherapy for early-stage non-small-cell

Cite this article as: $\mathrm{Xu} \mathrm{L}, \mathrm{Li} \mathrm{C}, \mathrm{Lu} \mathrm{H}$. Invasive mucinous adenocarcinoma of the lung. Transl Cancer Res 2019;8(8):29242932. doi: $10.21037 /$ tcr.2019.11.02 lung cancer: American Society of Clinical Oncology Endorsement of the American Society for Radiation Oncology Evidence-Based Guideline. J Clin Oncol 2018;36:710-9.

50. Ettinger DS, Wood DE, Akerley W, et al. NCCN guidelines insights: non-small cell lung cancer, version 4. 2016. J Natl Compr Canc Netw 2016;14:255-64.

51. Cha YJ, Kim HR, Lee HJ, et al. Clinical course of stage $\mathrm{IV}$ invasive mucinous adenocarcinoma of the lung. Lung Cancer 2016;102:82-8.

52. Mitsudomi T, Yatabe Y. Mutations of the epidermal growth factor receptor gene and related genes as determinants of epidermal growth factor receptor tyrosine kinase inhibitors sensitivity in lung cancer. Can Cancer Sci 2007;98:1817-24.

53. Jones MR, Lim H, Shen $Y$, et al. Successful targeting of the NRG1 pathway indicates novel treatment strategyfor metastatic cancer. Ann Oncol 2017;28:3092-7.

54. Lin G, Li H, Kuang J, et al. Acinar-Predominant Pattern Correlates With Poorer Prognosis in Invasive Mucinous Adenocarcinoma of the Lung. Am J Clin Pathol 2018;149:373-8. 